\title{
Criminologie
}

\section{Mesure de résultats des travaux communautaires chez les jeunes contrevenants : l'I.É.R.T.C.}

\section{Suzanne Carrier, Daniel Fortin et Danielle Paquet}

Volume 31, numéro 2, automne 1998

La sécurité privée

URI : https://id.erudit.org/iderudit/017421ar

DOI : https://doi.org/10.7202/017421ar

Aller au sommaire du numéro

\section{Éditeur(s)}

Les Presses de l'Université de Montréal

ISSN

0316-0041 (imprimé)

1492-1367 (numérique)

Découvrir la revue

Citer cet article

Carrier, S., Fortin, D. \& Paquet, D. (1998). Mesure de résultats des travaux communautaires chez les jeunes contrevenants : l'I.É.R.T.C. Criminologie, 31(2), 111-132. https://doi.org/10.7202/017421ar
Résumé de l'article

The community work program is designed for young offenders between the ages of 12 and 17. Participants are allowed to make restitution for their delinquent acts by working a certain number of hours in a neighborhood organization. Aside from this work aspect, the program also aims at arriving at various short-term results that serve as indications of the program's impact on participants. The lack of instruments able to systematically collect data for this purpose led to the creation of the Community Work Evaluation Tool (CWET). Grounded in the objectives of the community work program, the CWET measures the process by which its 13 items were arrived at, its validation and its psychometric properties. The CWET is argued to be a valid tool for evaluating the effects of such a program. These effects fall along four areas: (1) the personal evolution of each participant; (2) the participant's commitment to the completion of the program; (3) relationships between participants; and (4) the participant's attitude and openness toward the community. 
MESURE DE RESULTATS DES TRAVAUX COMMUNAUTAIRES CHEZ LES JEUNES CONTREVENANTS : l'I.É.R.T.C.'

Suzanne Carrier ${ }^{2}$

Daniel Fortin ${ }^{2}$

Danielle Paquet ${ }^{3}$

The community work program is designed for young offenders between the ages of 12 and 17. Participants are allowed to make restitution for their delinquent acts by working a certain number of hours in a neighborhood organization. Aside from this work aspect, the program also aims at arriving at various short-term results that serve as indications of the program's impact on participants. The lack of instruments able to systematically collect data for this purpose led to the creation of the Community Work Evaluation Tool (CWET). Grounded in the objectives of the community work program, the CWET measures the process by which its 13 items were arrived at, its validation and its psychometric properties. The CWET is argued to be a valid tool for evaluating the effects of such a program. These effects fall along four areas: (1) the personal evolution of each participant; (2) the participant's commitment to the completion of the program; (3) relationships between participants; and (4) the participant's attitude and openness toward the community.

\section{INTRODUCTION}

En vertu de la Loi sur les jeunes contrevenants, le programme juvénile de travaux communautaires s'adresse aux adolescents qui se sont rendus responsables d'infractions ou d'actes criminels. Il permet aux jeunes de réparer leur geste en s'engageant bénévolement, pendant un nombre d'heures prédéterminé, dans un organisme de leur communauté. Un organisme de

1. Ce projet est réalisé grâce à une subvention des Services aux collectivités de l'Université du Québec à Montréal.

2. Département de psychologie, Laboratoire de recherche en écologie humaine et sociale (LAREHS), Université du Québec à Montréal, C.P. 8888, Succursale centre-ville, Montréal (Québec), H3C 3P8.

3. Trajet-Jeunesse, Organisme de justice alternative, 1335, Mont-Royal, est, Montréal (Québec), H2J 1 Y6. 
justice alternative assure la mise en œuvre des travaux communautaires et le suivi des jeunes au cours de la réalisation de la mesure. Les jeunes sont référés par les délégués des centres de protection de l'enfance et de la jeunesse suite à la signature d'une mesure de rechange ou à une ordonnance de la cour du Québec, chambre de la jeunesse.

L'avènement et l'implantation de travaux communautaires, au cours de la décennie 1970, ont déjà été le sujet de plusieurs travaux (Simon et Vallières, 1981 ; Bernard, 1982 ; Carrier, 1982 ; Rizcalla et Deslandes, 1982 ; Bruneau, Laflamme et Linteau, 1984 ; Martinez, 1984 ; Caputo, 1987 ; Whitehead et $\mathrm{Lab}, 1989$ ). Ces écrits, dont la plupart sont de nature théorique, font ressortir un ensemble de points concernant les fondements des travaux communautaires. Les auteurs précisent que les travaux communautaires s'inscrivent dans une volonté de déjudiciarisation, c'est-à-dire que leur but est de fournir une alternative à la détention de courte durée dans les cas où l'intérêt public n'est pas mis en danger. Cette mesure s'appuie sur le désir de rendre les jeunes contrevenants responsables de leurs actes en leur permettant de réparer leur délit. Les travaux communautaires reposent sur le principe voulant que la communauté offre une solution plus humaine et plus susceptible d'accroitre les chances de réinsertion des jeunes contrevenants dans la société. Ce programme s'inscrit dans un effort de réhabilitation qui amène les jeunes à réfléchir sur eux-mêmes et sur leur conduite, tout en leur permettant d'entreprendre une démarche de réconciliation avec le monde des adultes.

L'examen de la documentation concernant les travaux communautaires révèle la rareté des études empiriques, lesquelles se limitent généralement à une description des caractéristiques des participants. Bien que les mesures de travaux communautaires existent depuis plusieurs années, il n'existe aucune évaluation systématique de leurs résultats auprès des jeunes. De plus, une brève enquête auprès des organismes de justice alternative montre l'absence d'outils pour la réalisation d'une collecte systématique de données à cette fin. Un instrument, comportant des items basés sur les objectifs fixés avec les jeunes participants aux travaux communautaires, est indispensable aux organismes de justice alternative voulant procéder à une analyse des résultats de ce programme. De plus, il procure une cohérence dans l'évaluation même si celle-ci est réalisée par des intervenants différents. L'Instrument d'évaluation de résultats des travaux communautaires (I.É.R.T.C.), présenté dans cet article, veut combler ce besoin. Construit à partir des objectifs des travaux communautaires, il permet une évaluation, par les intervenants, du fonctionnement des jeunes engagés dans ce programme. Plus particulièrement, l'I.É.R.T.C. rend possible une évaluation de la progression des jeunes entre le moment de leur arrivée et celui de leur sortie, de même que de l'atteinte des objectifs fixés. 
Après une présentation du cadre théorique autour duquel s'articule la construction de l'I.É.R.T.C., cet article décrit la méthodologie utilisée pour la constitution des items de l'instrument. Les items sont ensuite présentés, ainsi que les résultats de l'analyse qui vérifie la fidélité de l'instrument. Des normes, constituées à partir d'un indice du fonctionnement global de chaque jeune, sont également fournies. Enfin, la dernière section de l'article discute de l'intérêt mais aussi des limites de l'I.É.R.T.C. pour apprécier les résultats des travaux communautaires.

\section{CADRE THÉORIQUE}

La réalisation complète de la mesure par le jeune ne peut véritablement permettre, à elle seule, d'évaluer l'impact des travaux communautaires sur les participants, bien qu'elle en soit un critère de réussite essentiel. Par ailleurs, l'absence de récidive ne peut rendre compte de l'ensemble des effets des travaux communautaires sur les jeunes. Cet objectif ultime constitue un indicateur réducteur et controversé de l'efficacité des mesures pour jeunes délinquants (Lajoie et LeBlanc, 1990). En effet, pour connaître l'impact des travaux communautaires sur les adolescents qui y participent, il importe de considérer les résultats immédiats visés par le programme. Sur ce plan, l'examen de la documentation spécialisée révèle plusieurs objectifs qui peuvent servir d'indicateurs des effets du programme sur les participants. Tels que présentés dans les lignes qui suivent, ils se regroupent autour de quatre zones d'impacts : l'évolution du participant, l'engagement dans la réalisation de la mesure, les relations avec l'entourage et l'ouverture à la communauté.

\section{L'évolution du participant}

Dans les écrits spécialisés, une première zone d'impacts des travaux communautaires concerne l'évolution personnelle du participant face à ses actes délinquants et face à lui-même. Une série d'objectifs a trait à une responsabilisation du jeune face à l'acte délictueux commis. Ceux-ci sont d'abord formulés en termes de prise de conscience, par le participant, des conséquences de son infraction (Paquet, Choronzey, Lafortune, Marsolais et Ruiz, 1983), du tort causé (MSSS, 1993) et de l'impact de ses gestes (Filion, Giguère, Paquet et Simard, 1988 ; ROOQ, 1993). Les travaux communautaires visent à faire en sorte que l'adolescent assume la responsabilité de son délit (MSSS, 1993) et cela en lui permettant de réparer symboliquement les dommages causés (Collette-Carrière, 1986; Filion et al., 1988) et de se réconcilier en payant sa dette à la société (Paquet et al., 1983).

Outre la responsabilisation du jeune face au délit, la participation aux travaux communautaires vise une amélioration de ses habiletés sociales 
(ROOQ, 1992), de la perception qu'il a de lui-même (MSSS, 1993), ainsi qu'une valorisation à travers un travail positif lui permettant d'affirmer ses capacités personnelles (ROOQ, 1993). La satisfaction personnelle et la prise de conscience de ses propres possibilités sont, selon Collette-Carrière (1986), des acquis importants reliés à la réalisation d'une mesure de travaux communautaires.

\section{L'engagement dans la réalisation de la mesure}

Qu'il s'agisse d'une mesure de rechange ou d'une ordonnance du tribunal, une catégorie d'objectifs des travaux communautaires porte sur l'engagement du jeune dans la réalisation de la mesure. L'intervention auprès du jeune vise à ce qu'il adhère au programme selon les modalités de fonctionnement établies, qu'il réalise ses engagements (Paquet $e t$ al., 1983) et acquière le sens des responsabilités, notamment en respectant les horaires et les consignes de travail (Filion et al., 1988). De plus, certains jeunes constatent que la mesure permet de se sensibiliser à un métier ou à une profession, d'être valorisé dans un rôle d'aidant, de se sentir utile et de découvrir un autre sens au travail que le gain d'argent (Paquet $e$ t al., 1983).

\section{Les relations avec l'entourage}

Le rétablissement du lien social est une retombée positive des mesures de rechange (MSSS, 1993). En effet, le processus bilatéral par lequel le lien entre le jeune et son milieu est restauré favorise une réconciliation du jeune avec son entourage (Filion $e t$ al., 1988). D'abord, par les efforts qu'il déploie au sein de l'organisme participant, le jeune a un apport positif à son entourage (Collette-Carrière, 1986). Cela contribue à démystifier la délinquance et les jeunes contrevenants (Filion et al., 1988) et à rétablir, chez l'adolescent, une image sociale souvent ternie (ROOQ, 1992). De plus, la participation d'un jeune à une mesure de travaux communautaires lui fournit, entre autres, l'occasion d'établir des relations significatives avec un superviseur au sein de l'organisme d'accueil, de se découvrir un nouveau groupe d'appartenance et de se sensibiliser à la réalité de gens plus désavantagés que lui (Paquet et al., 1983).

\section{L'ouverture à la communauté}

La mesure de travaux communautaires contribue à préserver ou à renforcer le lien de l'adolescent avec sa communauté, laquelle est représentée par l'organisme (ROOQ, 1993). Les travaux communautaires visent à favoriser le service réel à la communauté par un travail utile et respectueux des besoins des organismes locaux (Filion et al., 1988). En ce sens, la proximité du milieu de vie du jeune est un critère important dans le choix de 
l'organisme d'accueil (Collette-Carrière, 1986). En démontrant au jeune qu'il a une place ou un rôle à jouer dans son milieu, le travail communautaire accroît son sentiment d'appartenance et son intégration dans la communauté (Paquet et al., 1983 ; Collette-Carrière, 1986 ; ROOQ, 1992). De plus, la mesure permet une ouverture du jeune sur la collectivité et la découverte de ressources communautaires répondant à des besoins précis de la population (Paquet et al., 1983 ; Filion et al., 1988).

Ainsi, le programme de travaux communautaires comporte une série de résultats immédiats qui sont indicateurs de quatre zones d'impacts auprès du jeune : l'évolution du participant, l'engagement dans la réalisation de la mesure, les relations avec l'entourage et l'ouverture à la communauté. Afin de permettre d'apprécier les résultats des travaux communautaires sur les jeunes participants, les items composant l'I.É.R.T.C. touchent ces quatre dimensions, émergentes à l'intérieur du processus de construction de l'instrument. Toutefois, avant de présenter les items de l'instrument, la méthodologie suivie pour le développement de l'I.É.R.T.C. est précisée dans la section suivante.

\section{MÉTHODOLOGIE}

Le développement de l'I.É.R.T.C. résulte d'un travail conjoint entre le chercheur et le personnel d'un organisme de justice alternative. Ce processus est constitué de trois étapes principales : l'élaboration des items inclus dans l'instrument, l'expérimentation de l'I.É.R.T.C. et la vérification de sa qualité psychométrique.

\section{Le constitution des items de l'I.E.R.T.C.}

Puisque l'I.É.R.T.C. est une mesure des résultats des travaux communautaires pour les participants, les items de l'instrument sont constitués à partir des objectifs visés, par le programme, chez les jeunes. Dans un premier temps, un inventaire des objectifs est effectué par le biais d'une consultation du personnel de l'organisme participant à l'étude, ainsi que par l'examen de documents disponibles à ce sujet. Au cours de cette étape, 59 objectifs sont répertoriés. Une liste de ces objectifs est soumise à chacun des intervenants, qui jugent de son exhaustivé.

Dans un deuxième temps, pour permettre au personnel de procéder à la classification des objectifs répertoriés en un nombre réduit d'items, la cartographie de concepts (Trochim, 1989 ; Dagenais et Bouchard, 1995a, 1995b) est utilisée. Pour ce faire, une pile de 59 fiches correspondant chacune à un des 59 objectifs de la liste est remise à chaque intervenant au cours d'une réunion. Individuellement, chaque intervenant regroupe les énoncés en 
catégories mutuellement exclusives, selon qu'ils sont, à son avis, conceptuellement rapprochés ou non. Les résultats sont inscrits dans une matrice de similarité, indiquant la présence ou l'absence d'association entre deux objectifs. Au moyen d'un traitement statistique combinant l'échelonnage multidimensionnel et l'analyse typologique hiérarchique, les objectifs que les participants considèrent comme associés sont réunis en grappes. Il en résulte une carte qui représente graphiquement ces regroupements d'objectifs. À partir de chacun de ceux-ci, un item de l'instrument est constitué. La figure 1 montre la carte obtenue pour la constitution des items de l'I.É.R.T.C. À titre d'exemple, l'îlot motivation face à la mesure, situé dans la partie droite de la carte, est constitué de la combinaison des objectifs 2 (s'impliquer activement dans l'organisation, le déroulement et le suivi de la mesure), 12 (respecter les modalités de la mesure et ses engagements face à l'organisme) et 41 (manifester de la motivation à compléter la mesure, à persévérer malgré les difficultés rencontrées).

Figure 1

Représentation cartographique des objectifs des travaux communautaires

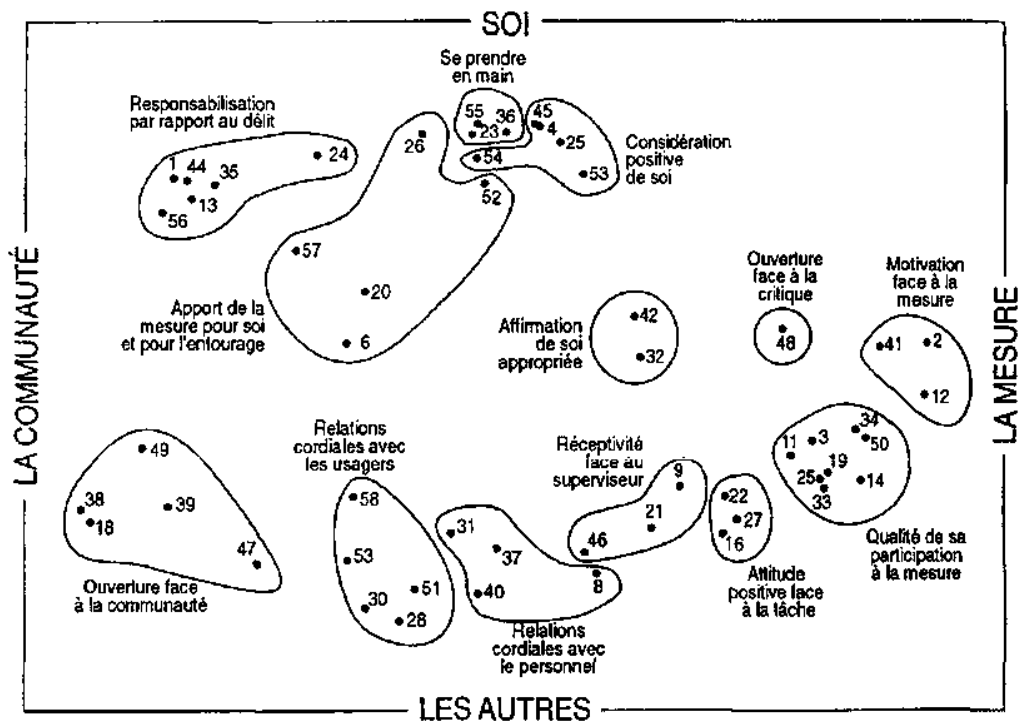

Dans le cas de l'I.É.R.T.C., cette procédure permet de dégager 13 regroupements d'objectifs qui correspondent chacun à un item de l'instrument. 
Les six premiers comportent une mesure à l'arrivée du jeune, au moment de déterminer les objectifs poursuivis par le jeune dans le cadre de la mesure, et à la sortie. Les sept items subséquents ne peuvent être l'objet d'une évaluation à l'arrivée; ils sont complétés au moment de fixer les objectifs et lors de la sortie.

Préalablement à une expérimentation de l'instrument, la vérification de la représentativité des 13 items est effectuée. Outre l'accord de la plupart des utilisateurs sur les items (Nunnally, 1978), la consultation d'experts contribue à assurer la validité de contenu de l'instrument (Anastasi, 1988). Ainsi, 16 partenaires locaux qui collaborent avec l'organisme pour la planification et le suivi de travaux communautaires sont invités à s'exprimer sur la capacité des items à rendre compte des résultats visés par le programme auprès des jeunes. Il s'agit de deux juges, d'un procureur de la couronne, d'un avocat de la défense, de deux délégués à la jeunesse, de cinq intervenants rattachés à un organisme de justice alternative et de cinq superviseurs de travaux communautaires.

Une fois confirmée la validité de contenu de l'instrument, un comité d'intervenants spécifie, avec la supervision du chercheur, des niveaux d'atteinte de l'objectif correspondant à chacun des items. À cette fin, le comité s'inspire de la méthode de développement d'échelles d'atteinte des buts (Kiresuk et Sherman, 1968 ; Kiresuk et Garwick, 1975 ; Cardillo et Smith, 1994). Bien que cette méthode ne soit pas initialement conçue pour apprécier des résultats individuels à partir d'objectifs qui seraient communs à l'ensemble des participants au programme, Kiresuk et Garwick (1975) indiquent qu'il peut être utile, pour la tenue des dossiers, de préciser des problèmes types pouvant être traduits en items communs pour tous les clients, comme c'est le cas pour l'I.É.R.T.C. La procédure de spécification des niveaux d'atteinte des objectifs, suggérée par Kiresuk et Garwick (1975), propose l'élaboration d'items comportant cinq niveaux mutuellement exclusifs, lesquels représentent le continuum des résultats observables par rapport à un but. Ainsi, pour chaque item de l'I.É.R.T.C., les niveaux -2 et 2 correspondent respectivement au pire et au meilleur des résultats qui peuvent être obtenus par les jeunes, tandis que le niveau 0 indique la performance la plus susceptible d'être visée ou atteinte par la majorité d'entre eux. Les différents niveaux de chacun des items de l'I.É.R.T.C. se caractérisent par des signes observables.

L'I.É.R.T.C. comporte, enfin, un indice traduisant le fonctionnement d'ensemble de chaque participant. Il correspond au total des scores obtenus sur les 13 items, à la sortie. 


\section{L'expérimentation de l'I.É.R.T.C. et l'échantillon}

Une fois déterminés les items et les modalités d'utilisation de l'instrument, 12 intervenants de l'organisme expérimentent l'I.É.R.T.C. Ceux-ci sont jumelés et, ainsi, chaque expérience de travaux communautaires est appréciée par deux juges indépendants en vue d'obtenir un indice du degré d'accord inter-juges. Dans tous les cas, le juge A est l'intervenant qui, au sein de l'organisme, a la responsabilité du suivi du jeune. Ainsi, un même intervenant peut faire office de juge A pour l'évaluation de certains jeunes et agir à titre de juge $\mathrm{B}$, pour d'autres jeunes. Ces derniers sont évalués à toutes les étapes des travaux communautaires, c'est-à-dire à leur arrivée, lors de la fixation des objectifs, et à la sortie. Le jugement est représenté par une cote individuelle basée sur le contact des juges avec le jeune depuis son arrivée jusqu'à la fin de la mesure.

Sur une période d'un an, l'I.É.R.T.C. est expérimenté pour le suivi, par les intervenants de l'organisme, de tous les jeunes engagés dans une mesure individuelle de travaux communautaires. Au total, sur une période d'un an, 108 expériences de travaux communautaires individuelles sont suivies, dont 81 menées à terme et évaluées. Les travaux communautaires réalisés par les participants à l'étude s'inscrivent dans le cadre d'une mesure de rechange $(33,0 \%)$ ou d'une ordonnance du tribunal $(67,0 \%)$. Le délai moyen entre le délit et la signature de la mesure de rechange ou l'ordonnance du tribunal est respectivement de 113,35 jours $(s=50,55)$ et de 221,84 jours $(s=157,37)$. En moyenne, les travaux communautaires débutent après 70,68 jours $(\mathrm{s}=$ 47,48 ) après la signature de la mesure de rechange ou l'ordonnance du tribunal. Bien que le nombre moyen d'heures prescrites soit de 31,85 heures ( $s=16,95$ ), il y a, en moyenne, un délai de 33,67 jours $(s=34,01)$ entre le début et la fin de la mesure.

La majorité des participants $(89,9 \%)$ sont des garçons. Les jeunes sont, en moyenne, âgés de 16,52 ans $(s=1,41)$. Ceux provenant d'une famille monoparentale sont plus nombreux $(54,1 \%$ ) que ceux dont les parents vivent ensemble $(29,4 \%)$. Tandis que $17,4 \%$ des participants déclarent être sans occupation, $64,7 \%$ des jeunes sont encore aux études. Les types de délits se répartissent comme suit : $45,9 \%$ contre la propriété, $22,9 \%$ contre la personne et $10,1 \%$ contre le système judiciaire. À cela s'ajoute $21,1 \%$ des cas qui présentent une combinaison des types de délits.

\section{La vérification de la qualité psychométrique de l'instrument}

La vérification de la qualité psychométrique de l'instrument est réalisée par une série d'analyses statistiques. Elle consiste d'abord en une analyse des items, puis en une étude de la fidélité de l'I.É.R.T.C. 


\section{L'analyse des items}

Suite à l'expérimentation de l'I.É.R.T.C., une analyse est effectuée à partir des scores octroyés par les juges pour chacun des items de l'instrument. À cette fin, quatres analyses sont réalisées :

- premièrement, l'examen de l'occurrence des valeurs de -2 à 2 ayant une fréquence nulle est révélateur de la pertinence des niveaux de chaque item et cela, à l'arrivée, au moment de déterminer les objectifs, et à la sortie ;

- deuxièmement, la vérification du postulat voulant que le niveau zéro représente le résultat le plus susceptible d'être visé ou atteint par la majorité des sujets est effectuée par un calcul de la moyenne des scores octroyés au moment de spécifier l'objectif sur chaque échelle. De façon complémentaire, le calcul de la moyenne des scores obtenus à la sortie permet de connaître jusqu'à quel point le niveau zéro correspond au résultat obtenu par la majorité ;

- troisièmement, la sensibilité des items réfère à leur capacité de rendre compte de l'effet du programme de travaux communautaires sur la progression du jeune. Elle est mesurée par une série de test t. Ceux-ci vérifient, sur les six premiers items, si les scores à la sortie sont plus élevés que ceux donnés à l'arrivée et si cette différence est significative ;

- quatrièmement, la fidélité inter-juge porte sur la similitude des scores alloués, pour chaque item et à chaque moment de mesure, par les juges $\mathrm{A}$ comparativement aux juges $\mathbf{B}$. Le test $t$ pour données appariées, ainsi que le coefficient de corrélation de Pearson sont utilisés pour examiner la fidélité inter-juge. De plus, un calcul du pourcentage des scores attribués par les juges avec un écart supérieur à 1 est effectué.

\section{L'indice de fonctionnement global}

La fidélité inter-juges de l'indice de fonctionnement global est vérifiée par un calcul du coefficient de corrélation de Pearson. Également, le coefficient alpha de Cronbach permet d'examiner la cohérence interne de l'indice.

\section{RÉSULTATS}

\section{Les items de l'I.É.R.T.C.}

L'I.É.R.T.C. comprend 13 items comportant chacun 5 niveaux. Ils sont présentés en annexe.

\section{La pertinence des niveaux}

L'examen des valeurs ayant une fréquence nulle est révélatrice de la pertinence des niveaux de chacun des items. À l'arrivée, tous les niveaux 
sont utilisés par l'un ou l'autre juge. Lors de la détermination des objectifs, les niveaux -2 et -1 sont plus rarement sélectionnés. Cela est cohérent avec le fait que les travaux communautaires visent un progrès chez les participants et que, de plus, le niveau 0 est théoriquement considéré comme le résultat le plus probable. Enfin, à la sortie, pour 5 des 13 échelles, le niveau -2 n'est pas utilisé, ainsi qu'il est indiqué dans les colonnes minimum et maximum du tableau 1.

Lorsque l'on considère globalement les scores octroyés à l'arrivée, lors de la détermination des objectifs, et à la sortie, tous les niveaux sont utilisés, à un moment ou l'autre, pour tous les items, à l'exception de deux items. Il s'agit des items 9 (considération positive de soi) et 12 (relations cordiales avec la clientèle de l'organisme). Pour ces deux items, les juges utilisent quatre niveaux. Ainsi, dans l'ensemble, les cing niveaux de l'instrument s'avèrent utiles, que ce soit à l'arrivée, au moment de déterminer les objectifs, ou à la sortie.

\section{Le niveau zéro et les scores octroyés à chaque moment de mesure}

Le niveau zéro correspond à un niveau d'attente théorique, représentant le résultat théoriquement le plus susceptible d'être visé et atteint par la majorité des participants. En conformité avec ce postulat, les objectifs fixés et les scores à la sortie, pour chaque item, doivent correspondre idéalement à une moyenne de 0 .

Dans le cas de l'I.É.R.T.C, au moment de fixer les objectifs, la moyenne des scores octroyés pour l'ensemble des items est de, 75 pour les juges A et de, 73 pour les juges $B$. Elle se situe légèrement au-dessus du niveau 0 . Les scores, au moment de déterminer les objectifs des items 4 et 5 , sont ceux qui s'écartent le plus du niveau 0 ; ils sont, en moyenne, supérieurs à 1 . Afin d'accroître la validité de l'instrument, le niveau zéro pour ces deux items est revisé de manière à ce que les résultats visés s'en approchent davantage.

À l'instar des scores donnés au moment de fixer les objectifs, les scores attribués à la sortie sont en moyenne légèrement supérieurs à zéro. En effet, la moyenne des scores alors octroyés par les juges $\mathrm{A}$ est de, 74 et de, 78 lorsqu'il s'agit des juges B.

\section{La sensibilité de l'instrument}

L'instrument est sensible s'il permet de détecter un changement en comparant les résultats obtenus à l'arrivée et ceux à la sortie, pour les 6 premiers items de l'instrument, lesquels comportent ces deux moments de mesure. Dans le cas de ces 6 items, tous les scores à la sortie sont plus élevés que les 
scores attribués à l'arrivée, et cette différence est significative dans tous les cas.

Bien qu'il ne s'agisse pas en soi de la sensibilité de l'instrument, une comparaison des scores octroyés au moment de déterminer l'objectif avec ceux donnés à la sortie est effectuée. Il n'y a pas de différence significative pour 9 des 13 items de l'instrument, indiquant que les juges fixent des objectifs qui s'approchent des résultats atteints.

Tableau 1

Accord inter-juges des scores octroyés à la sortie

\begin{tabular}{|c|c|c|c|c|c|c|c|c|c|}
\hline \multirow[b]{2}{*}{$\begin{array}{l}\text { numéro } \\
\text { de l'item }\end{array}$} & \multirow[b]{2}{*}{$\begin{array}{l}\text { mini- } \\
\text { mum }\end{array}$} & \multirow[b]{2}{*}{$\begin{array}{l}\operatorname{maxi}- \\
\text { mum }\end{array}$} & \multicolumn{2}{|c|}{ Juge A } & \multicolumn{2}{|c|}{ Juge B } & \multirow[b]{2}{*}{$t^{*}$} & \multirow[b]{2}{*}{$\mathrm{r}^{* *}$} & \multirow[b]{2}{*}{$\begin{array}{c}\% \text { des } \\
\text { scores } \\
\text { attribués } \\
\text { avec } \\
\text { écart > } \\
1 * * *\end{array}$} \\
\hline & & & moyenne & $\begin{array}{l}\text { écart- } \\
\text { type }\end{array}$ & moyenne & $\begin{array}{l}\text { écart- } \\
\text { type }\end{array}$ & & & \\
\hline 1 & -2 & 2 &, 36 & .98 &, 26 & .86 & 1,21 & .68 & 6,3 \\
\hline 2 & -2 & 2 &, 92 & 1,11 &, 83 & 1,11 &, 90 & .70 & 7,2 \\
\hline 3 & -2 & 2 &, 52 & 1,13 &, 58 & 1,09 &,- 66 &, 79 & 2.8 \\
\hline 4 & -1 & 2 & .97 & .77 &, 82 &, 76 & 1,69 & .70 & 1,3 \\
\hline 5 & -1 & 2 &, 71 & 1,15 &, 72 & 1,04 &,- 13 & .71 & 8.4 \\
\hline 6 & -2 & 2 &, 63 & 1,05 &, 60 & 1,02 &, 32 &, 73 & 4,6 \\
\hline 7 & -2 & 2 & 1,00 & 1,15 & 1,06 & 1,06 & -.67 &, 72 & 7,2 \\
\hline 8 & -2 & 2 & 35 & 1,07 & 1,13 &, 13 &, 80 &, 72 & 9.9 \\
\hline 9 & -1 & 2 & .49 &, 95 &, 52 &, 84 &,- 46 &, 67 & 6.1 \\
\hline 10 & -1 & 2 & 1,05 &, 99 & 1,04 &, 89 &, 15 &, $7 \mathbf{i}$ & 3,6 \\
\hline 11 & -2 & 2 &, 90 & 1,00 &, 97 &, 97 &,- 84 &, 75 & 2,8 \\
\hline 12 & -1 & 2 &, 92 &, 92 & .86 &, 86 & .62 &, 75 & 5,0 \\
\hline 13 & -2 & 2 &, 86 & 1,09 & .80 & 1,18 & .84 &, 84 & 2,4 \\
\hline
\end{tabular}

* non significatif pour l'ensemble des 13 objectifs

** $\mathrm{p} \leqslant .001$ pour l'ensembie des 13 objectifs

**** \% des jeunes chez qui l'écart des scores octroyés à la sortie par le juge A et le juge B est supérieur à 1

\section{La fidélité inter-juges}

La fidélité inter-juges porte sur la similitude des scores alloués, à chaque moment de mesure, par les juges A comparativement aux juges B. Le 
tableau 1 présente les données relatives à la fidélité inter-juges pour les scores à la sortie. Le tableau 1 montre également la moyenne et l'écart-type pour les juges $A$ et $B$, ainsi que la valeur du test $t$ indiquant qu'il n'y a pas de différence significative entre les résultats octroyés par ceux-ci. De plus, pour tous les items, un coefficient de corrélation de Pearson significatif est obtenu entre les scores donnés par les juges A et B, comme le montre le tableau 1. Les coefficients de corrélation obtenus se situent entre ,67 et ,84. Enfin, les pourcentages des individus, pour lesquels l'écart des valeurs attribuées par les juges est supérieur à 1 , varient de $1,3 \%$ à $9,9 \%$. L'ensemble de ces analyses indiquent un degré de fidélité inter-juges acceptable, bien que moyennement élevé.

\section{L'indice de fonctionnement global}

Une fidélité inter-juges élevée est obtenue pour l'indice de fonctionnement global $(\mathrm{r}=, 91 ; \mathrm{p}<.001)$, basé sur la somme des 13 scores octroyés à la sortie. Pour les scores donnés par les juges A, l'alpha standardisé est de ,93 avec une corrélation inter-items moyenne de ,52. Dans le cas des juges B, l'alpha standardisé est de, 93 avec une corrélation inter-items moyenne de , 51 .

\section{Les normes}

Les normes de l'I.É.R.T.C. sont établies à partir de l'indice de fonctionnement global. Le tableau 2 fournit les scores bruts et les scores $\mathrm{Z}$ correspondant. Il indique aussi les points de coupure entre les déciles.

\section{DISCUSSION}

Dans le cadre d'une évaluation de résultats, l'I.É.R.T.C. permet de vérifier efficacement les effets immédiats des travaux communautaires sur les jeunes participants. La fiabilité de l'instrument tient d'abord à sa validité de contenu, reconnue par les partenaires locaux de l'organisme consultés à ce sujet. Cela confirme l'importance d'examiner les résultats immédiats des travaux communautaires pour les jeunes, plutôt que de considérer uniquement l'accomplissement du nombre d'heures prescrites ou, à plus long terme, l'absence de récidive. Afin de favoriser la représentativité du contenu, la cartographie de concepts s'avère un procédé avantageux pour constituer des items à partir de la représentation que les intervenants se font des objectifs visés par le programme. La carte conceptuelle obtenue, à partir de laquelle les items de l'I.É.R.T.C. sont constitués, s'organise autour de quatre zones d'impacts des travaux communautaires sur les jeunes: soi, la mesure, les autres et la communauté. Émergeant lors de la construction de l'instrument, 
CHEZ LES JEUNES CONTREVENANTS : L'I.É.R.T.C.

Tableau 2

Table des normes de l'indice de fonctionnement global de l'I.E.R.T.C.

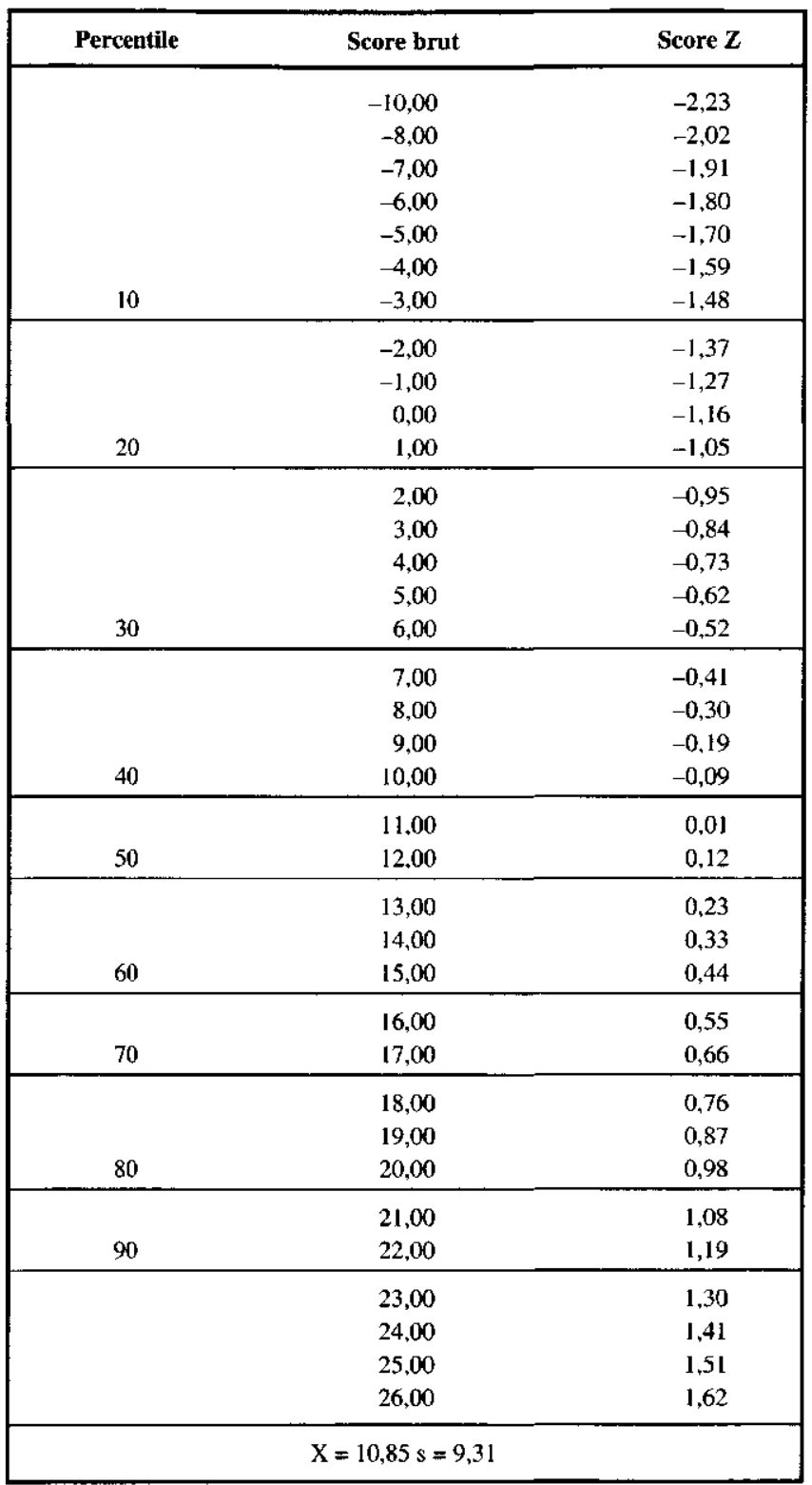


ces catégories s'apparentent à celles dégagées lors de la recension des écrits spécialisés présentée au début de l'article.

Outre la validation du contenu, l'analyse des items, réalisée suite à l'expérimentation, confirme, dans l'ensemble, la pertinence des 5 niveaux reproduits pour chaque item. Les juges utilisent les 5 niveaux lorsqu'ils évaluent les jeunes, à l'exception de deux items où les valeurs -2 ne sont jamais octroyées.

En ce qui a trait plus particulièrement à l'utilisation du niveau 0 , au moment de fixer les objectifs et à la sortie, la moyenne des scores octroyés pour l'ensemble des items se situe légèrement au-dessus du niveau 0 , qui correspond théoriquement au résultat le plus susceptible d'être atteint par la majorité des participants. Les items 4 et 5 sont améliorés, après l'expérimentation, pour que les objectifs déterminés en rapport avec ceux-ci s'approchent davantage de 0 .

Les items de I'I.É.R.T.C. présentent une bonne sensibilité ainsi qu'une fidélité inter-juges acceptable. Sur ce dernier aspect, il y a absence de différences significatives entre les résultats, suffisamment corrélés, accordés par les juges $\mathbf{A}$ et $\mathbf{B}$.

Enfin, l'indice de fonctionnement global, qui résume la participation du jeune à la mesure, possède une cohérence interne élevée pour chacun des juges et est l'objet d'une corrélation inter-juges élevée.

Même si les analyses réalisées au cours de cette étude démontrent la fiabilité de l'I.É.R.T.C., la validité de l'instrument peut être améliorée par une expérimentation subséquente engageant plusieurs organismes de justice alternative et, dès lors, un nombre plus considérable d'intervenants et de jeunes. Cela peut aussi permettre l'obtention de normes ajustées à une population plus vaste.

Dans la perspective d'une évaluation de résultats des travaux communautaires, l'I.É.R.T.C. ne touche qu'un volet, celui des effets du programme chez les jeunes. Les travaux communautaires étant basés sur une mise à contribution de partenaires locaux, il peut devenir également important de considérer les effets du programme sur la communauté participante. Enfin, outre les travaux communautaires individuels, les organismes de justice alternative assurent d'autres programmes destinés aux jeunes contrevenants : amélioration des aptitudes sociales, travaux compensatoires, mesures jumelées. Une évaluation de résultats de ces programmes requiert le développement d'instruments conçus à partir de leurs objectifs particuliers, distincts de ceux mesurés par l'I.É.R.T.C. 


\section{RÉFÉRENCES}

ANASTASI, A. (1988), Psychological Testing (6 édition), New York, Macmillan.

BERNARD, L. (1982), La déjudiciarisation juvénile par l'article 40 de la Loi 24 : les enjeux d'un conflit entre le social et le judiciaire, Université de Montréal, Montréal, mémoire de maîtrise inédit.

BRUNeAU, M., LAFLAMME, M. et LiNTEAU, M.-J. (1984), Programme de responsabilisation des jeunes contrevenants et de leurs communautés face à la déliquance : Devis d'évaluation inédit, Centre de services sociaux de Québec, Québec.

CAPUTO, T.C. (1987), « The Young Offender Act : Children's rights, children's wrongs », Canadian Public Policy, vol. 13, p. 125-143.

CARDILLO, J. E. et SMITH, A. (1994), «Psychometric Issues », in T. J. KIRESUK, A. SMITH et J. E. CARDILLO (dir.), Goal Attainment Scaling : Application, Theory and Measurement, (p. 173-212), Hillsdale, Lawrence Erlbaum Associates.

CARRIER, N. (1982), Les mesures alternatives à l'incarcération, une responsabilitét nécessairement partagée, Congrès des substituts au procureur général, Québec.

COLLETTE-CARRIÈRE, R. (1986), Les travaux communautaires jeunesse : une expérimentation québécoise, Centre de consultation de la région de Québec, Solliciteur Général du Canada, Ottawa.

CRONBACH, L. J. (1988), « Five perspectives on validity argument », in H. WAINER et H. I. BRAUN (dir.), Test Validity, (p. 3-17), Hillsdale, New Jersey, Lawrence Erlbaum Associates.

DAGENAIS, C. et BOUCHARD, C. (1995a), "Concept mapping in the context of modelization of intervention with families in crisis », In J. J. HOX, B. F. VAN DER MEULEN, J. M. JANSSENS, J. J. F. TER LAAK et L. W. C. TAVECCHIO (dir.), Advances in Family Research, (p. 217-228), Amsterdam, Thesis Publishers.

DAGENAIS, C. et BOUCHARD, C. (1995b), « La cartographie de concepts dans le contexte de la modélisation des interventions auprès de familles en crise ", Les cahiers d'analyse du GRAVE, vol. 2 et 3, p. 1-22.

FILION, D., GIGUÈRE, G., PAQUET, D. et SIMARD, A. (1988), L'organisme orienteur : sa philosophie et sa pratique d'intervention : guide de référence, Regroupement des organismes orienteurs du Québec, Montréal.

KIRESUK, T. et GARWICK, G. (1975), Basic Goal Attainment Scaling Procedures, Minnepolis, Minnesota Program Evaluation Project.

KIRESUK, T. J. et SHERMAN, R. E. (1968), « Goal attainment scaling : a general method for evaluating comprehensive community health programs ", Community Mental Health Journal, vol. 4, p. 433-453.

LAJOIE, J. et LEBLANC, M. (1990), « Débat autour des mesures pour jeunes délinquants », Criminologie, vol. XXIII, $\mathrm{n}^{\circ}$ 2, p. 107-123.

MARTINEZ, J. (1984), Évaluation du programme de travail communautaire JeunesseLongueuil, Centre des services sociaux Richelieu, Longueuil.

MINISTĖRE DE LA SANTÉ ET DES SERVICES SOCIAUX (1993), Loi sur les jeunes contrevenants : manuel de référence, Gouvernement du Québec, Ministère de la santé et des services sociaux, direction de l'adaptation sociale, Québec.

NUNNALLY, J. C. (1978), Psychometric Theory, New-York, Mc Graw-Hill Book Compagny. 
PAQUET, D.. CHORONZEY, C., LAFORTUNE, M., MARSOLAIS, M. et RUIZ, P. (1983), Le programme de travaux communautaires : expérimentation d'une mesure alternative pour les jeunes, Programme de travaux communautaires, Montréal.

REGROUPEMENT DES ORGANISMES ORIENTEURS DU QUÉBEC (1992), Les organismes orienteurs du Québec : cadre de référence, Regroupement des organismes orienteurs du Québec, Montréal.

REGROUPEMENT DES ORGANISMES ORIENTEURS DU QUÉBEC (1993), Document de réflexion sur le cadre d'intervention d'un organisme orienteur, Regroupement des organismes orienteurs du Québec, Montréal.

RIZKALLA, S. et DESLANDES, P. (1982), Les sentences de travaux communautaires: leur passé, leur présent et leur futur, Rapport des colloques organisés par la Société de criminologie du Québec, Montréal, Québec, Chicoutimi.

SIMON, H. et VALLIÈRES, S. (1981), L'évaluation d'une nouvelle mesure pénale : les travaux communautaires au Québec, École de criminologie de l'Université de Montréal, Montréal.

TROCHIM, W. M. K. (1989), « An introduction to concept mapping for planning and evaluation ", Evaluation and Program Planning, vol. 12, p. 1-16.

WHITEHEAD, J.T. et LAB, S.P. (1989), «A Meta-analysis of juvenile correctional treatment », Jourmal of Research In Crime And Delinquency, vol. 26, p. 276-295. 
Annexe

Les échelles de l'I.É.R.T.C.

\begin{tabular}{|c|c|c|c|c|c|c|}
\hline & n.a. & -2 & -1 & 0 & +1 & +2 \\
\hline $\begin{array}{l}\text { 1. Responsabilisation par } \\
\text { rapport au délit }\end{array}$ & & $\begin{array}{l}\text { - ne se reconnaît pas } \\
\text { responsable de son délit, } \\
\text { done ne reconnaît pas le } \\
\text { préjudice causé, la } \\
\text { dimension réparatrice de } \\
\text { la mesure, n'accepte pas } \\
\text { la conséquence de son } \\
\text { délit. }\end{array}$ & $\begin{array}{l}\text { - se reconnaît comme } \\
\text { responsable de son délit : } \\
\text { - accepte lat conséquence } \\
\text { de son délit et fou se } \\
\text { conforme à la } \\
\text { conséquence. }\end{array}$ & $\begin{array}{l}\text { - se reconnaît comme } \\
\text { responsable de son délit ; } \\
\text { - accepte la conséquence } \\
\text { de son délit; } \\
\text { - a l'intention de ne plus } \\
\text { récidiver. }\end{array}$ & $\begin{array}{l}\text { - se reconnaît comme } \\
\text { responsable de son délit ; } \\
\text { - accepte la conséquence } \\
\text { de son délit : } \\
\text { - a l'intention de ne plus } \\
\text { récidiver ; } \\
\text { - reconnaît le préjudice } \\
\text { causé. }\end{array}$ & $\begin{array}{l}\text { - se reconnaît comme } \\
\text { responsable de son délit ; } \\
\text { - accepte la conséquence } \\
\text { de son délit ; } \\
\text { - a l'intention de ne plus } \\
\text { récidiver ; } \\
\text { - reconnaît le préjudice } \\
\text { causé : } \\
\text { - fait le lien entre ses } \\
\text { problèmes et ses agirs } \\
\text { délinquants sans } \\
\text { chercher à se disculper ; } \\
\text { - saisit la dimension } \\
\text { réparatrice de la mesure. }\end{array}$ \\
\hline $\begin{array}{l}\text { 2. Motivation face à la } \\
\text { mesure }\end{array}$ & & $\begin{array}{l}\text { - ne respecte pas les } \\
\text { modalités de la mesure et } \\
\text { ses engagements face à } \\
\text { l'organisme. }\end{array}$ & $\begin{array}{l}\text { - a de la difficulté à } \\
\text { respecter les modalités } \\
\text { de la mesure et ses } \\
\text { engagements face à } \\
\text { l'organisme. }\end{array}$ & $\begin{array}{l}\text { - respecte les modalités } \\
\text { de la mesure et ses } \\
\text { engagements face à } \\
\text { l'organisme. }\end{array}$ & $\begin{array}{l}\text { - respecte les modalités } \\
\text { de la mesure et ses } \\
\text { engagements face à } \\
\text { l'organisme ; } \\
\text { - manifeste de la } \\
\text { motivation à compléter la } \\
\text { mesure, à persévérer } \\
\text { malgré les difficultés } \\
\text { rencontrées. }\end{array}$ & $\begin{array}{l}\text { - respecte les modalités } \\
\text { de la mesure et ses } \\
\text { engagenents face à } \\
\text { l'organisme ; } \\
\text { - manifeste de la } \\
\text { motivation à compléter la } \\
\text { mesure, à persévérer } \\
\text { malgré les difficultés } \\
\text { rencontrées ; } \\
\text { - s'engage et collabore } \\
\text { activement dans } \\
\text { l'organisation, le } \\
\text { déroulement et le suivi } \\
\text { de la mesure. }\end{array}$ \\
\hline
\end{tabular}


Annexe (suite)

\begin{tabular}{|c|c|c|c|c|c|c|}
\hline & n.a. & $-\mathbf{2}$ & -1 & 0 & +1 & +2 \\
\hline 3. Se prendre en main & & $\begin{array}{l}\text { - est incapable de faire } \\
\text { une rétlexion sur sa } \\
\text { façon de faire des choix. }\end{array}$ & $\begin{array}{l}\text { - hésite à faire de } \\
\text { nouveaux choix et à } \\
\text { changer ses habitudes de } \\
\text { vie. }\end{array}$ & $\begin{array}{l}\text { - amorce une réflexion } \\
\text { sur sa façon de faire des } \\
\text { choix; } \\
\text { - discerne les choix } \\
\text { pouvant lui être } \\
\text { bénéfiques de ceux } \\
\text { pouvant lui être } \\
\text { nuisibles. }\end{array}$ & $\begin{array}{l}\text { - amorce une réflexion } \\
\text { sur sa façon de faire des } \\
\text { choix ; } \\
\text { - discerne les choix } \\
\text { pouvant lui être } \\
\text { bénéfiques de ceux } \\
\text { pouvant lui être } \\
\text { nuisibles; } \\
\text { - affirme vouloir changer } \\
\text { ses habitudes de vie. }\end{array}$ & $\begin{array}{l}\text { - amorce une réflexion } \\
\text { sur sa façon de faire des } \\
\text { choix ; } \\
\text { - discerne les choix } \\
\text { pouvant lui être } \\
\text { bénéfiques de ceux } \\
\text { pouvant lui être } \\
\text { nuisibles; } \\
\text { - affïrme vouloir changer } \\
\text { ses habitudes de vie ; } \\
\text { - identifie des moyens } \\
\text { concrets afin de se } \\
\text { réaliser (ex. : retour à } \\
\text { l'école, stage en milieu } \\
\text { de travail ; démarche } \\
\text { d'aide ou de thérapie; } \\
\text { bénévolat). }\end{array}$ \\
\hline 4. Ouverture à la critique & & $\begin{array}{l}\text { - réagit négativement à } \\
\text { la critique (de façon } \\
\text { agressive). }\end{array}$ & $\begin{array}{l}\text { - écoute les critiques } \\
\text { mais essaie de se } \\
\text { disculper ou de se défiler. }\end{array}$ & $\begin{array}{l}\text { - écoute les critiques } \\
\text { qu'on lui exprime. }\end{array}$ & $\begin{array}{l}\text { - se montre très réceptif } \\
\text { aux critiques qu'on lui } \\
\text { exprime. }\end{array}$ & $\begin{array}{l}\text { - fait des efforts pour se } \\
\text { réajuster en tenant } \\
\text { compte des critiques } \\
\text { qu'on lui adresse. }\end{array}$ \\
\hline $\begin{array}{l}\text { 5. Affirmation de soi } \\
\text { appropriée }\end{array}$ & & $\begin{array}{l}\text { - prend sa place } \\
\text { inadéquatement (être en } \\
\text { retruil ou être } \\
\text { envahissant). }\end{array}$ & $\begin{array}{l}\text { - a de la difficulté à } \\
\text { cemer la place qu'il doit } \\
\text { prendre (tendance à être } \\
\text { en retrait ou envahissant). }\end{array}$ & $\begin{array}{l}\text { - exprime adéquatement } \\
\text { son opinion lorsque } \\
\text { nécesaire ; } \\
\text { - prend sa place de façon } \\
\text { adéquate. }\end{array}$ & $\begin{array}{l}\text { - exprime adéquatement } \\
\text { son opinion lorsque } \\
\text { nécessaire ; } \\
\text { - prend sa place, se fait } \\
\text { respecter et s'affirme de } \\
\text { façon adéquate. }\end{array}$ & $\begin{array}{l}\text { - exprime adéquatement } \\
\text { son opinion lorsque } \\
\text { nécessaire ; } \\
\text { - se fait respecter, } \\
\text { s'affirme et prend sa } \\
\text { place de façon adéquate ; } \\
\text { - s'affirme adéquatement } \\
\text { dans des situations où il } \\
\text { n'est pas aisé de le faire. }\end{array}$ \\
\hline
\end{tabular}


Annexe (suite)

\begin{tabular}{|c|c|c|c|c|c|c|}
\hline & n.a. & -2 & -1 & 0 & +1 & +2 \\
\hline $\begin{array}{l}\text { 6. Ouverture face à la } \\
\text { communauté }\end{array}$ & & $\begin{array}{l}\text { - ne démontre aucun } \\
\text { intérêt ou sensibilité face } \\
\text { aux problèmes sociaux } \\
\text { vécus dans sa } \\
\text { communauté et aux } \\
\text { efforts des organismes } \\
\text { pour y remédier. }\end{array}$ & $\begin{array}{l}\text { - connaît déjà ou prend } \\
\text { connaissance des } \\
\text { services offerts dans sa } \\
\text { communauté mais ne s'y } \\
\text { intéresse que très peu. }\end{array}$ & $\begin{array}{l}\text { - connaît déjà ou prend } \\
\text { connaissance des } \\
\text { services offerts dans sa } \\
\text { communauté et s'y } \\
\text { intéresse. }\end{array}$ & $\begin{array}{l}\text { - connaît déjà ou prend } \\
\text { connaissance des } \\
\text { services offerts dans sa } \\
\text { communauté et s'y } \\
\text { intéresse ; } \\
\text { - développe une attitude } \\
\text { positive face au } \\
\text { bénévolat. }\end{array}$ & $\begin{array}{l}\text { - connaît déjà ou prend } \\
\text { connaissance des } \\
\text { services offerts dans sa } \\
\text { communauté et s'y } \\
\text { intéresse ; } \\
\text { - développe une attitude } \\
\text { positive face au } \\
\text { bénévolat : } \\
\text { - se sensibilise aux } \\
\text { problèmes vécus par les } \\
\text { gens de sa communauté ; } \\
\text { - développe un sentiment } \\
\text { d'appartenance face à sa } \\
\text { communauté ; } \\
\text { - démontre de l'interêt } \\
\text { face aux activités et à la } \\
\text { clientèle de l'organisme. }\end{array}$ \\
\hline $\begin{array}{l}\text { 7. Qualité de sa } \\
\text { participation à la mesure }\end{array}$ & & $\begin{array}{l}\text { - cherche à négocier } \\
\text { continuellement de } \\
\text { nouvelles ententes; } \\
\text { - quantité et qualité de } \\
\text { travail insatisfaisantes: } \\
\text { - se présente quand il } \\
\text { veut ; } \\
\text { - est un poids pour } \\
\text { l'organisme. }\end{array}$ & $\begin{array}{l}\text { - nécessite un } \\
\text { encadrement soutenu ; } \\
\text { - arrive souvent en retard } \\
\text { ou } \\
- \text { s'absente souvent mais, } \\
\text { une fois sur place, offre } \\
\text { une bonne qualité et } \\
\text { quantité de travail ; } \\
\text { ou } \\
\text { - est ponctuel et assidu } \\
\text { mais inconstant dans sà } \\
\text { qualité de travail. }\end{array}$ & $\begin{array}{l}\text { - se montre serviable, } \\
\text { disponible, coopérant ; } \\
\text { - ne cherche pas à } \\
\text { renégocier } \\
\text { continuellement de } \\
\text { nouvelles ententes ; } \\
\text { - nécessite un } \\
\text { encadrement minimal (en } \\
\text { termes de soutien, } \\
\text { d'encouragement et de } \\
\text { rappel) pour fournir un } \\
\text { rendement satisfaisant : } \\
\text { - foumit, de façon } \\
\text { constante, une quantité } \\
\text { de travail satisfaisante. }\end{array}$ & \begin{tabular}{|l|} 
- se montre serviable, \\
disponible, coopérant ; \\
- ne cherche pas à \\
renégocier \\
continuellement de \\
nouvelles ententes; \\
- nécessite un \\
encadrement minimal (en \\
termes de soutien, \\
d'encouragement et de \\
rappel) pour fournir un \\
rendement salisfaisant ; \\
- fournit, de façon \\
constante. une quantité \\
de travail satisfaisante ; \\
- est ponctuel et assidu.
\end{tabular} & $\begin{array}{l}\text { - se montre serviable, } \\
\text { disponible, coopérant; } \\
\text { - ne cherche pas à } \\
\text { renégocier } \\
\text { continuellement de } \\
\text { nouvelles ententes ; } \\
\text { - nécessite un } \\
\text { encadrement minimal (en } \\
\text { termes de soutien, } \\
\text { d'encouragement et de } \\
\text { rappel) pour fournir un } \\
\text { rendement satisfaisant ; } \\
\text { - fournit, de façon } \\
\text { constante, une quantité } \\
\text { de travail satisfaisante : } \\
\text { - est ponctuel et assidu; } \\
\text { - offre un travail de } \\
\text { bonne qualité de façon } \\
\text { constante. }\end{array}$ \\
\hline
\end{tabular}


Annexe (suite)

\begin{tabular}{|c|c|c|c|c|c|c|}
\hline & n.a. & -2 & -1 & 0 & +1 & +2 \\
\hline $\begin{array}{l}\text { 8. Apport de la mesure } \\
\text { pour soi et pour } \\
\text { l'entourage }\end{array}$ & & $\begin{array}{l}\text { - ne fait aucune réflexion } \\
\text { critique concernant } \\
\text { l'apport de la mesure } \\
\text { pour soi et pour } \\
\text { l'entourage. }\end{array}$ & $\begin{array}{l}\text { - surćvalue ou sous- } \\
\text { évalue son apport à } \\
\text { l'organisme. }\end{array}$ & $\begin{array}{l}\text { - découvre l'utilité que } \\
\text { peuvent avoir ses actions } \\
\text { pour son entourage : } \\
\text { - évalue sa contribution à } \\
\text { l'organisme. }\end{array}$ & $\begin{array}{l}\text { - découvre l'utilité que } \\
\text { peuvent avoir ses actions } \\
\text { pour son entourage : } \\
\text { - évalue sa contribution à } \\
\text { l'organisme ; } \\
\text { - reconnait ce qu'il retire } \\
\text { de la mesure ; } \\
\text { - considère la mesure } \\
\text { comme formatrice. }\end{array}$ & $\begin{array}{l}\text { - découvre l'utilité que } \\
\text { peuvent avoir ses actions } \\
\text { pour son entourage ; } \\
\text { - Évalue sa contribution à } \\
\text { l'organisme; } \\
\text { - reconnaît ce qu'il retire } \\
\text { de la mesure ; } \\
\text { - considère la mesure } \\
\text { comme formatrice ; } \\
\text { - a une infuence positive } \\
\text { sur son milieu (amis, } \\
\text { famille, etc). }\end{array}$ \\
\hline $\begin{array}{l}\text { 9. Considération positive } \\
\text { de soi }\end{array}$ & & $\begin{array}{l}\text { - ne reconnaît pas ses } \\
\text { habiletés et ses forces. }\end{array}$ & $\begin{array}{l}\text { - reconnaît difficilement } \\
\text { ses habiletés et ses } \\
\text { forces. }\end{array}$ & $\begin{array}{l}\text { - reconnaît ses habiletés } \\
\text { et ses forces. }\end{array}$ & $\begin{array}{l}\text { reconnaît ses habiletés, } \\
\text { ses forces et ses limites; } \\
\text { - se découvre de } \\
\text { nouvelles aptitudes : } \\
\text { - se découvre de } \\
\text { nouveaux champs } \\
\text { d'intérêt. }\end{array}$ & $\begin{array}{l}\text { - reconnaît ses habiletés, } \\
\text { ses forces et ses limites; } \\
\text { - se découvre de } \\
\text { nouvelles aptitudes ; } \\
\text { - se découvre de } \\
\text { nouveaux champs } \\
\text { d'intérêt ; } \\
\text { - adopte une vision et } \\
\text { une attitude positive face } \\
\text { à son avenir ; } \\
\text { - acquiert une meilleure } \\
\text { estime de soi. }\end{array}$ \\
\hline
\end{tabular}


Annexe (suite)

\begin{tabular}{|c|c|c|c|c|c|c|}
\hline & n.a. & -2 & -1 & 0 & +1 & +2 \\
\hline $\begin{array}{l}\text { 10. Réceptivité face au } \\
\text { superviseur }\end{array}$ & & $\begin{array}{l}\text { - est fermé aux conseils } \\
\text { de son superviseur ; } \\
\text { - est méfiant et } \\
\text { méprisant à l'égard de } \\
\text { son superviseur. }\end{array}$ & $\begin{array}{l}\text { - est réceptif aux } \\
\text { conseils de son } \\
\text { superviseur sans } \\
\text { toutefois pouvoir les } \\
\text { traduire de façon } \\
\text { tangible : } \\
\text { - est maladroit dans sa } \\
\text { façon de s'exprimer ou } \\
\text { de négocier avec son } \\
\text { superviseur. }\end{array}$ & $\begin{array}{l}\text { - se montre réceptif aux } \\
\text { conseils de son } \\
\text { superviseur. }\end{array}$ & $\begin{array}{l}\text { - se montre réceptif aux } \\
\text { conseils de son } \\
\text { superviseur ; } \\
\text { - s'exprime } \\
\text { adéquaternent avec son } \\
\text { superviseur. }\end{array}$ & $\begin{array}{l}\text { - se montre réceptif aux } \\
\text { conseils de son } \\
\text { superviseur; } \\
\text { - s'exprime } \\
\text { adéquatement avec son } \\
\text { superviseur; } \\
\text { - développe une relation } \\
\text { positive avec son } \\
\text { superviseur (confiance } \\
\text { face à l'adulte, ouverture, } \\
\text { sincérité). }\end{array}$ \\
\hline $\begin{array}{l}\text { 11. Relations cordiales } \\
\text { avec le personnel }\end{array}$ & & $\begin{array}{l}\text { - fait preuve d'itrespect } \\
\text { et d'impatience à l'égard } \\
\text { du personnel de } \\
\text { l'organisme et ne fournit } \\
\text { pas d'effort pour } \\
\text { améliorer ces rapports. }\end{array}$ & $\begin{array}{l}\text { - manifeste de } \\
\text { l'indifférence ou de la } \\
\text { froideur à l'égard du } \\
\text { personnel. }\end{array}$ & $\begin{array}{l}\text { - foumit les efforts } \\
\text { requis, at besoin. pour } \\
\text { améliorer ses rapports } \\
\text { avec le personnel de } \\
\text { l'organisme; } \\
\text { - manifeste du respect } \\
\text { envers le personnel. }\end{array}$ & $\begin{array}{l}\text { - fournit les efforts } \\
\text { requis, au besoin, pour } \\
\text { améliorer ses rapports } \\
\text { avec le personnel de } \\
\text { l'organisme : } \\
\text { - manifeste du respect } \\
\text { envers le personnel ; } \\
\text { - dévełoppe de bonnes } \\
\text { relations avec le } \\
\text { personnel de } \\
\text { l'organisme. }\end{array}$ & $\begin{array}{l}\text { - fournit les efforts } \\
\text { requis, au besoin, pour } \\
\text { améliorer ses rapports } \\
\text { avec le personnel de } \\
\text { l'organisme ; } \\
\text { - manifeste du respect } \\
\text { envers le personnel ; } \\
\text { - développe de bonnes } \\
\text { relations avec le } \\
\text { personnel de } \\
\text { l'organisme : } \\
\text { - gagne la confiance du } \\
\text { personnel de } \\
\text { l'organisme. }\end{array}$ \\
\hline
\end{tabular}


Annexe (suite)

\begin{tabular}{|c|c|c|c|c|c|c|}
\hline & n.a. & -2 & -1 & 0 & +1 & +2 \\
\hline $\begin{array}{l}\text { 12. Relations cordiales } \\
\text { avec la clientèle de } \\
\text { l'organisme }\end{array}$ & & $\begin{array}{l}\text { - fait preuve d'irrespect } \\
\text { ou d'impatience à l'égard } \\
\text { de la clientèle de } \\
\text { l'organisme. }\end{array}$ & $\begin{array}{l}\text { - manifeste de la } \\
\text { froideur ou de } \\
\text { l'indifférence à l'égard } \\
\text { de la clientèle de } \\
\text { l'organisme. }\end{array}$ & $\begin{array}{l}\text { - manifeste du respect } \\
\text { envers la clientèle de } \\
\text { l'organisme. }\end{array}$ & $\begin{array}{l}\text { - manifeste du respect } \\
\text { envers la clientèle de } \\
\text { l'organisme ; } \\
\text { - développe de bonnes } \\
\text { relations avec la clientèle } \\
\text { de l'organisme ; } \\
\text { - fournit les efforts } \\
\text { nécessaires pour } \\
\text { améliorer. au besoin, les } \\
\text { relations avec la clientèle } \\
\text { de l'organisme. }\end{array}$ & $\begin{array}{l}\text { - manifeste du respect } \\
\text { envers la clientèle de } \\
\text { l'organisme ; } \\
\text { - développe de bonnes } \\
\text { relations avec la clientèle } \\
\text { de l'organisme ; } \\
\text { - fournit les efforts } \\
\text { nécessaires pour } \\
\text { améliorer, au besoin, les } \\
\text { relations avec la clientèle } \\
\text { de l'organisme ; } \\
\text { - fait preuve de patience } \\
\text { et de dévouement à } \\
\text { l'égard de la clientèle de } \\
\text { l'organisme ; } \\
\text { - acquiert une } \\
\text { connaissance des } \\
\text { difficultés et de la réalité } \\
\text { propres à la clientèle de } \\
\text { l'organisme. }\end{array}$ \\
\hline $\begin{array}{l}\text { 13. Attitude positive face } \\
\text { à la tâche }\end{array}$ & & $\begin{array}{l}\text { - «fait du temps », } \\
\text { cherche à en faire le } \\
\text { moins possible. }\end{array}$ & $\begin{array}{l}\text { - s'acquitte de sa tâche } \\
\text { sans démontrer un intérêt } \\
\text { particulier. }\end{array}$ & $\begin{array}{l}\text { - démontre de l'intérêt et } \\
\text { de l'ouverture face aux } \\
\text { tâches à effectuer. }\end{array}$ & $\begin{array}{l}\text { - démontre de l'intérêt et } \\
\text { de l'ouverture face aux } \\
\text { tâches à effectuer ; } \\
\text { - fait preuve de } \\
\text { dynamisme face aux } \\
\text { tâches à effectuer. }\end{array}$ & $\begin{array}{l}\text { - démontre de l'intérêt et } \\
\text { de l'ouverture face aux } \\
\text { tâches à effectuer : } \\
\text { - fait preuve de } \\
\text { dynamisme face aux } \\
\text { tâches à effectuer ; } \\
\text { - fait preuve d'initiative } \\
\text { et đe débrouillardise face } \\
\text { aux tâches à effectuer. }\end{array}$ \\
\hline
\end{tabular}

